\title{
A SINFONI view of circum-nuclear star-forming rings in spiral galaxies
}

\author{
Jesús Falcón-Barroso ${ }^{1}$, Torsten Böker ${ }^{1}$, Eva Schinnerer ${ }^{2}$, \\ Johan H. Knapen ${ }^{3}$ and Stuart Ryder ${ }^{4}$ \\ ${ }^{1}$ European Space and Technology Centre, Keplerlaan 1, 2200 AG Noordwijk, The Netherlands \\ email: jfalcon@rssd.esa.int \\ ${ }^{2}$ Max-Planck Institut für Astronomie, Königstuhl 17, D-69117 Heidelberg, Germany \\ ${ }^{3}$ Instituto de Astrofísica de Canarias, Via Láctea s/n, E-38200 La Laguna, Spain \\ ${ }^{4}$ Anglo-Australian Observatory, PO Box 296, Epping, NSW1710, Australia
}

\begin{abstract}
We present near-infrared (H- and K-band) SINFONI integral-field observations of the circumnuclear star formation rings in five nearby spiral galaxies. We made use of the relative intensities of different emission lines (i.e. [FeII], HeI, $\mathrm{Br} \gamma$ ) to age date the stellar clusters present along the rings. This qualitative, yet robust, method allows us to discriminate between two distinct scenarios that describe how star formation progresses along the rings. Our findings favour a model where star formation is triggered predominantly at the intersection between the bar major axis and the inner Lindblad resonance and then passively evolves as the clusters rotate around the ring ('Pearls on a string' scenario), although models of stochastically distributed star formation ('Popcorn' model) cannot be completely ruled out.
\end{abstract}

Keywords. galaxies: spiral; galaxies: nuclei; galaxies: stellar content; galaxies: star clusters; galaxies: starburst; galaxies: individual (NGC 613, NGC 1079, NGC 1300, NGC 5248, IC 1438)

\section{Introduction}

Star-forming nuclear rings in the inner regions of early- and intermediate Hubble type (Sa-Sc) galaxies are one of the key pieces to understand the transport of gas to the nuclei of galaxies and therefore how secular evolution proceeds. The basic picture of how these rings form seems well established, both theoretically and observationally, and is usually associated to the interplay between bar-driven inflow and bar resonances (e.g. Schwarz 1984; Combes \& Gerin 1985; Athanassoula 1992; Knapen et al. 1995). A non-axisymmetric gravitational potential, nearly always due to the presence of a stellar bar or oval distortion, causes the gas to lose angular momentum and spiral in towards the nucleus. During the inflow, and because of its dissipative nature, the gas accumulates around the radii at which the stellar orbits experience dynamical resonances with the rotating bar potential. In the case of the nuclear rings discussed here this typically happens at the Inner Lindblad resonance. When observed in more detail, the gas enters the ring via two tightly wound spiral arms or dust lanes. At the contact points between the dust lanes and the ring, the gas becomes less turbulent, and enters the almost circular orbits delineating the ring. While it is clear that there is abundant (molecular) gas throughout the ring, there is some debate about how and where star formation occurs.

In this paper we present our efforts to understand the preferred model of star formation along the rings, based on SINFONI integral-field observations, of circumnuclear starforming rings in five nearby spiral galaxies (NGC 613, NGC 1079, NGC 1300, NGC 5248, IC 1438). We refer the reader to Böker et al. (2007) for a more complete discussion of the full sample. 

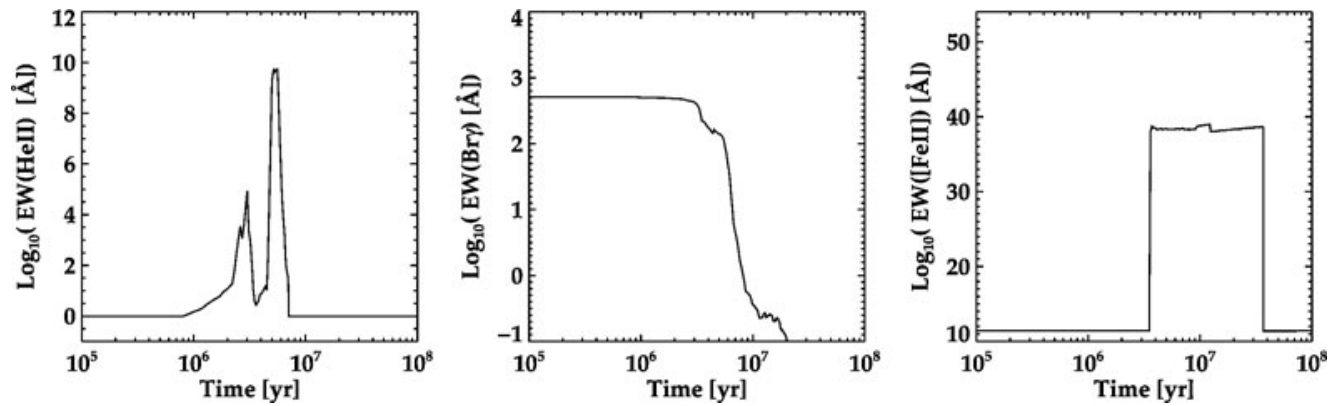

Figure 1. Evolution of the equivalent widths of $\mathrm{HeII}, \mathrm{Br} \gamma$ and $[\mathrm{FeII}]$ as a function of time for instantaneous STARBURST99 models. The maximum contribution of each of the three lines occurs in distinct, yet overlapping, time episodes. We use this property to produce a more accurate age dating of the stellar clusters along the star-forming rings (see text). Data shown here are for models with $\mathrm{z}=0.02$, an IMF slope $\alpha=2.35$, with an upper mass limit of $100 \mathrm{M}_{\odot}$. Models for other metallicities, IMF slopes and upper mass limit show similar behaviour.

\section{Measuring the stellar cluster ages}

Measuring the relative ages of the different stellar clusters (i.e. hotspots) along the ring is a difficult task. The orbital time scales in the ring are short (e.g. a few tens of Myr). In order to perform the age dating it is therefore imperative that one uses a tracer that it's sensitive to these time scales. The most widely used diagnostic for this purpose is the $\mathrm{H} \alpha$ emission line. There are several reasons why this line is a popular choice. First, it is an optical emission line that can be easily measured. Second, its equivalent width (EW) decreases almost monotonically with time for an instantaneous burst (see the STARBURST99 prediction, Leitherer et al. 1999) allowing us determine the evolution stage of a cluster depending of the EW value. The use of this emission line, however, is limited to clusters in the age range 3 to $10 \mathrm{Myr}$, and therefore any inferred age differences have to be small (few Myrs). Below $3 \mathrm{Myr}$ the EW of the $\mathrm{H} \alpha$ is almost constant, and above 10 Myr the value is well below typical detection limits $(\sim 1 \AA)$.

An alternative approach is to make use of several spectral lines, whose emission peak at different times. In our case we use the flux of three emission lines that are prominent in the NIR spectra of the star forming regions in our sample of galaxies: HeI, Br $\gamma$, and [FeII]. Under the assumption that the underlying bulge/disk can be considered quiescent, and that most of the emission is produced in the ring itself, line fluxes are a better probe than the EWs because they require an accurate knowledge of the continuum emission not only from the hot spot itself but also the underlying bulge and/or disk, which is very hard to measure. The HeI and $\operatorname{Br} \gamma$ lines are both produced by photo-ionization in the vicinity of O- or B-type stars. Given that the ionization energy for the HeI line is higher than that of $\mathrm{Br} \gamma$, it requires the presence of hotter and more massive stars, and hence its flux falls off more rapidly after an instantaneous burst than that of the $\mathrm{Br} \gamma$ line. The time range covered by these two lines is from 0 to $10 \mathrm{Myr}$. Larger ages can be probed with the [FeII] line (a tracer of fast shocks produced in supernova remnants) whose contribution is almost constant from 3 to $35 \mathrm{Myr}$, where it sharply decreases. With these three lines we are therefore able to probe ages in the range between 0 and $35 \mathrm{Myr}$, which represents a good match to the expected travel time of gas and star clusters around the ring. An illustration of their evolution with time (as predicted by STARBURST99) is presented in Figure 1. Note that in the figure HeII $(\lambda \lambda 4686 \AA)$ has been used as a surrogate for $\mathrm{HeI}$, and $[\mathrm{FeII}](\lambda \lambda 1.26 \mu \mathrm{m})$ for $[\mathrm{FeII}](\lambda \lambda 1.64 \mu \mathrm{m})$. 


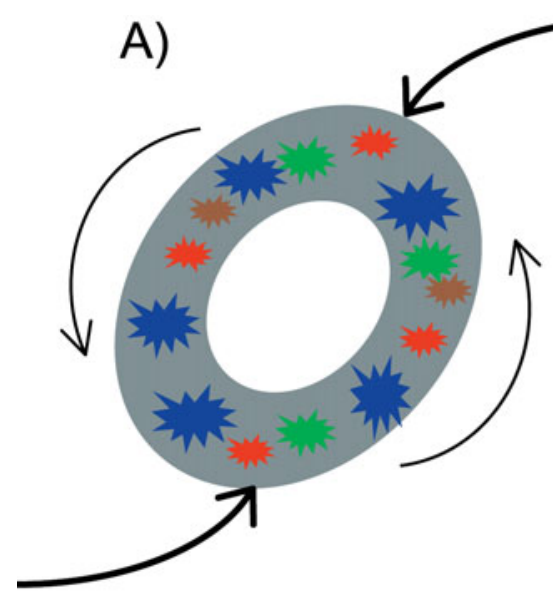

"Popcorn"

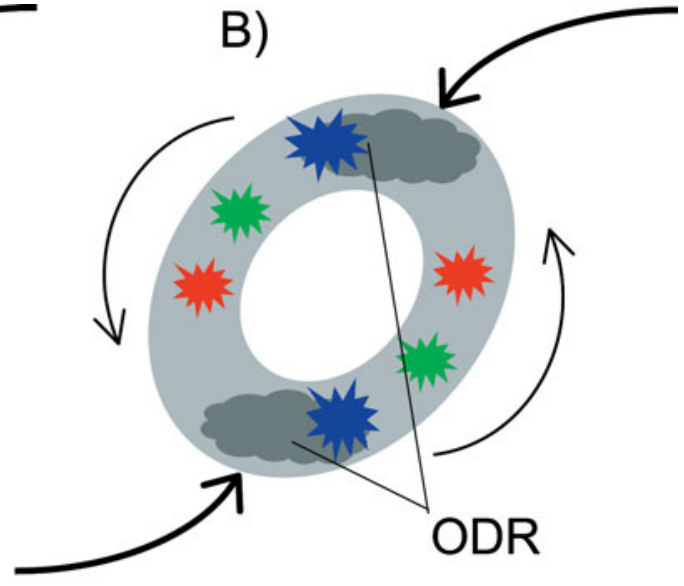

B)

"Pearls on a string"

Figure 2. Illustration of two proposed scenarios for the evolution of star formation in a nuclear ring. Dark grey areas denote dense, cold gas that is likely to lead to star formation. The various star symbols denote young stellar clusters, their colors reflect an increase in cluster age from blue to green to red. A clear age sequence is expected only in the 'Pearls on a string' scenario. Figure from Böker et al. (2007).

\section{The 'Popcorn' vs 'Pearls on a string' model scenarios}

In Figure 2 we illustrate two plausible mechanisms that describe how star-formation proceeds along the cirnumnuclear rings. In the first scenario, the 'Popcorn' model, gas enters the ring and accumulates around it with no preferred location. Once a critical density is reached, the gas becomes unstable to gravitational collapse and star formation is triggered. In this model, individual hot spots collapse at random times and locations within the ring, and therefore there is no systematic age sequence. In the figure (left plot) the different star bursts are denoted by the star symbols and the different colors indicate different star formation epochs. In the second scenario, Figure 2 (right), gas enters the ring at the intersection between the bar major axis and the inner Lindblad resonance. Downstream from this location, a point that we will call overdensity region (ODR), the gas density becomes sufficiently high to ignite star formation in a short-lived burst. A young cluster formed there will continue its orbit around the ring, but star formation will cease as soon as the first supernova explosions expel the gas. A series of starbursts triggered in the ODR will then produce a sequence of star clusters that enter the ring like 'Pearls on a string'. In this scenario, the star clusters should show a bi-polar age gradient around the ring, with the youngest clusters found close to the ODR, and increasingly older cluster ages in the direction of rotation, up to the opposite ODR. This is shown in the figure where the color sequence blue-green-red denotes clusters with increasing age.

In Figure 3 we put these two models to the test by showing the observations for NGC 613, one of the five galaxies in our sample. In the left panel we display a F666W HST image of the nuclear regions. The ring is oulined with an ellipse, and two star symbols mark the position of the ODRs (based on the regions with highest dust extinction in the ring). In the right panel we present a false-color image constructed from the SINFONI emission line maps of $\mathrm{HeI}, \mathrm{Br} \gamma$, and [FeII] assigned to the blue, green, and red color channels, respectively. The bottom half of the ring shows the trends expected under the 'Pearls on a string' picture: an age sequence (blue-green-red) in the different hotspots. 

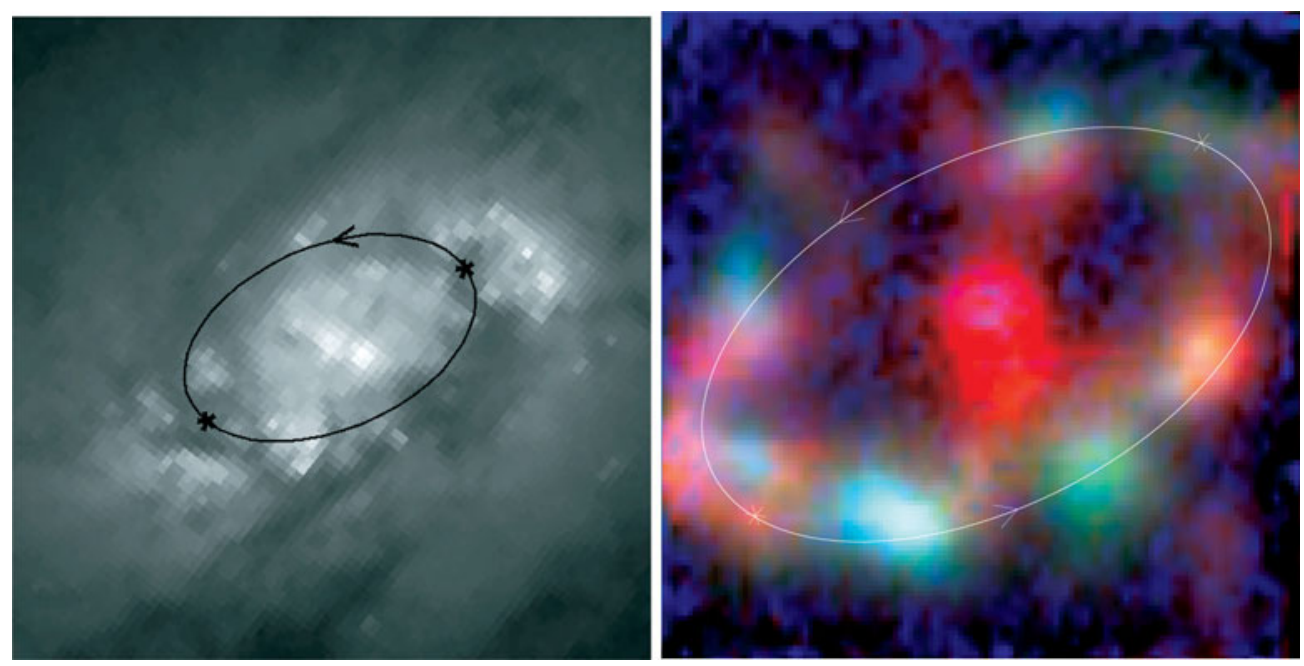

Figure 3. Left: Archival HST/WFPC2 F606W map of NGC 613. The outlined ellipse marks the location and sense of rotation (denoted with and arrow) of matter along the star-forming ring. Indicated are the assumed locations of the ODRs (marked with a star symbol) where infalling gas is compressed. Right: false color map of the emission line morphology in the inner 8 " $\times 8$ " of NGC 613. The blue channel represents HeI, green $\mathrm{Br} \gamma$, and red [FeII] emission. Note that at least in the southern half of the ring - the hottest stars (as marked by He I emission in blue) are located immediately downstream from the contact points. In contrast, the [FeII] emission in red occurd predominantly further along the ring, with $\mathrm{Br} \gamma$ emission dominated the region in between. This map supports the 'Pearls on a string' scenario, where star formation is triggered at the ODRs, forms stellar clusters than then age passively along their orbit. Figure from Böker et al. (2007).

The sequence is less obvious in the top part of the ring due to the strong interaction between the ring and the presence of a well-known radio jet (Hummel et al. 1987). In the whole sample, not shown here, three out of five galaxies show some evidence for an age gradient of hot spots along the ring, while the remaining two galaxies have incomplete information and thus are consistent with either model.

At this point our results are not totally conclusive, although they favour the 'Pearls on a string' model. Larger samples are needed to shed more light on this issue. Nevertheless, as demonstrated in this contribution, the proposed method of using NIR line ratios to estimate relative ages of young star clusters is a very powerful tool for this purpose.

\section{Acknowledgements}

JFB would like to thank the organizing committee and in particular Martin Bureau for a very fruitful and stimulating meeting.

\section{References}

Athanassoula, E. 1992, MNRAS, 259, 345

Böker, T., Falcón-Barroso, J., Schinnerer, E., Knapen, J. H., \& Ryder, S. 2007, AJ, submitted Combes, F. \& Gerin, M. 1985, A\&A, 150, 327

Hummel, E., Jörsäter, S., Lindblad, P. O., \& Sandqvist, A. 1987, A\& A, 172, 51

Knapen, J. H., Beckman, J. E., Heller, C. H., Shlosman, I., \& de Jong, R. S. 1995, ApJ, 454, 623

Leitherer, C., et al. 1999, ApJS, 123, 3

Schwarz, M. P. 1984, MNRAS, 209, 93 